\title{
Personalised multidisciplinary management for patients with chronic obstructive pulmonary disease (COPD)
}

Chronic obstructive pulmonary disease (COPD) is a major cause of mortality, morbidity (breathlessness, cough, impaired quality of life, infective exacerbations), health care costs (hospitalisations, emergency department visits, medication costs) and lost work productivity globally. Solutions to overcome this enormous burden of disease are urgently needed. In this fournal of Thoracic Disease Focused Issue on 'Advances in Multidisciplinary Care for COPD', the themes of diagnosis, pathogenesis, treatment and multimorbidities are comprehensively addressed from a multidisciplinary perspective. All of these themes build upon current preventive strategies in public health, to minimize exposure to tobacco smoke, air pollution, occupational dusts and other factors leading to COPD.

\section{Diagnosis}

Accurate and early detection of COPD sets the scene for appropriate management of this chronic lung condition. As chronic breathlessness can provide a diagnostic challenge in primary and tertiary care, Olivia Ferry and colleagues propose a diagnostic pathway with primary, secondary and tertiary level investigations for patients with chronic dyspnoea.

\section{Pathogenesis}

Understanding the complex pathogenesis of COPD provides the foundations for earlier diagnosis, biomarker development and targeted treatment. Mari Hikichi and colleagues discuss the basic pathogenesis of COPD, with specific focus on oxidative stress, altered immune response of the airway inflammatory cells, exaggerated cellular senescence of the lung structural cells, and cell death with expanded inflammation. Hannah O'Farrell and team members outline the potential role of extracellular vesicles, which are small membrane-bound vesicles released by nearly all cell types and contain bioactive cargo that are key mediators. Hannah O'Farrell and co-investigators report an original study of the clinical utility of multiple target quantitative polymerase chain reaction (qPCR) array to detect microbial pathogens in patients with COPD, during exacerbations and stability. Brielle Parris and co-authors provide insight into the common pathogenic links between COPD and lung cancer, and describe key molecular pathways that overlap in the development of these coexisting conditions. Annalicia Vaughan and co-authors summarise the nexus between the lungs and the gut— the gut-lung axis—and how dietary factors (including intake of fibre) can affect the gut microbiome, with implications for systemic and airway effects.

\section{Treatment}

Evidence-based approaches to the management of COPD are well-established in clinical guidelines. However, translation to clinical practice remains a challenge, with issues of barriers to health care access, complex health systems and variation in practice. Fanny Ko and co-authors offer a perspective on comprehensive care for patients with COPD by multidisciplinary teams, across the care continuum. Renae McNamara and colleagues present innovative strategies to improve the reach and engagement in pulmonary rehabilitation, to overcome barriers to access for this target population. Huib Kerstjens and coauthors summarise the principles of pharmacological management of patients with COPD, and describe recent developments and updates, especially in airway pharmacology. Hang Ding and colleagues review emerging evidence for the application of digital health—-telemonitoring, telehealth and mHealth—as a potential solution for improving COPD care at scale. Emily Sneath and co-authors review important pre-hospital and emergency department pathways of care for exacerbations of COPD, and demonstrate how important these systems are for acute care.

\section{Multimorbidities}

Patients with COPD present with multimorbidities which interact with the primary lung disease and require treatment. Peter Collins and co-authors provide an evidence update on nutritional support for patients with COPD, focusing on targeted multi- 
modal interventions to overcome weight loss, sarcopenia and cachexia. Marsus Pumar and co-investigators describe an RCT of cognitive behavioural therapy for patients with chronic lung disease and comorbid anxiety and/or depression symptoms.

Through the eyes of people living with 'COPD', for various reasons, they have 'copped' a chronic lung condition which affects their quality of life and has major implications for their health. By focusing on multidisciplinary care, we hope that this series of invited papers-from scientists and clinicians—will highlight new knowledge in the field of COPD care, make a call to action for creative health care solutions, and ultimately improve the lives of people with COPD.

\section{Acknowledgments}

We would like to acknowledge the patients and staff participating in our research studies, and funding from the National Health and Medical Research Council (NHMRC) and The Prince Charles Hospital Foundation.

\section{Footnote}

Conflicts of Interest: The authors have no conflicts of interest to declare.

Ethical Statement: The authors are accountable for all aspects of the work ensuring that questions related to the accuracy or integrity of all parts of the work are appropriately investigated and resolved.

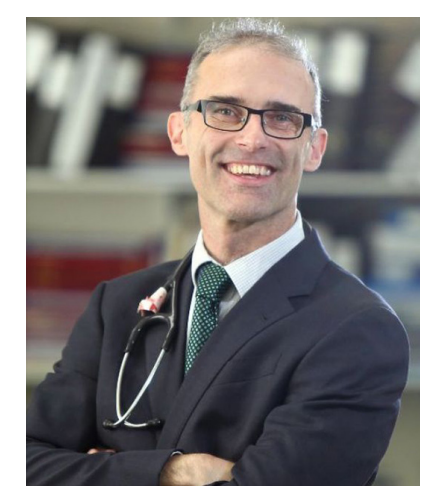

Henry M. Marshall

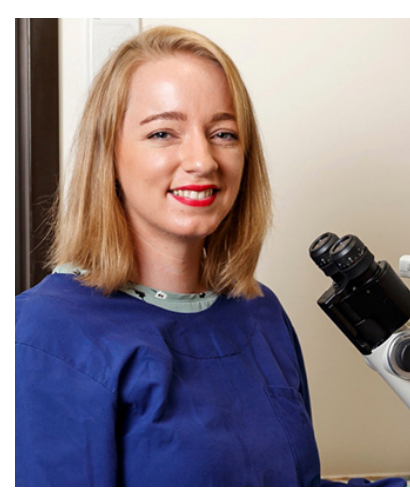

Annalicia Vaughan

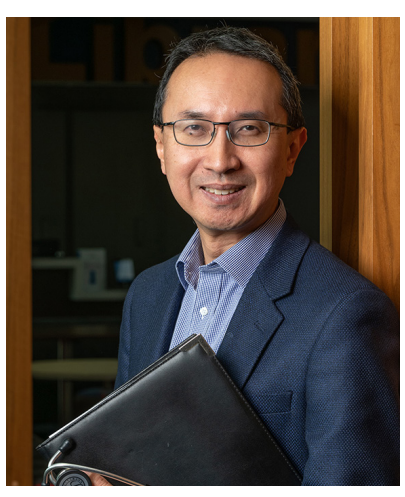

Ian A. Yang

\section{Henry M. Marshall \\ (Email: Henry.Marshall@health.qld.gov.au) \\ Annalicia Vaughan \\ (Email:a.vaughan@uq.edu.au) \\ Ian A. Yang \\ (Email:Ian.Yang@health.qld.gov.au)}

Thoracic Program, The Prince Charles Hospital, Metro North Hospital and Health Service, and UQ Thoracic Research Centre, Faculty of Medicine, The University of Queensland, Brisbane, Australia.

doi: $10.21037 /$ jtd.2019.10.52

View this article at: http://dx.doi.org/10.21037/jtd.2019.10.52

Cite this article as: Marshall HM, Vaughan A, Yang IA.

Personalised multidisciplinary management for patients with chronic obstructive pulmonary disease (COPD). J Thorac Dis 2019;11(Suppl 17):S2115-S2116. doi: 10.21037/jtd.2019.10.52 